Journal of Case Reports 2018;8(3):211-214

\title{
A Rare Case of a Synchronous Ovarian Tumor in a Young Girl
}

\author{
Manisha H Albal, Kanav P Kumar \\ Department of Pediatric Surgery, NKP Salve Institute of Medical Sciences \& Research Centre and Lata Mangeshkar Hospital, \\ Nagpur, Maharashtra, India.
}

\section{Corresponding Author: \\ Dr. Kanav P Kumar \\ Email: kanav.k92@gmail.com}

This is an Open Access article distributed under the terms of the Creative Commons Attribution License (creativecommons.org/ licenses/by/3.0).

Received

Accepted

Published

April 14, 2017

August 1,2018

September 10, 2018

\begin{abstract}
Background: Bilateral involvement of ovarian tumors is well known. Very few cases of synchronous bilateral primary ovarian tumors of different histologic subtypes are reported. Here we report a rare case of a left ovarian dysgerminoma with a right ovarian teratoma in an 11 year old girl. Case Report: Patient presented with abdominal pain since one month. A lump of size $15 \times 10 \mathrm{~cm}$ was occupying the whole abdomen. CECT abdomen showed a solid-cystic, heterogeneous mass arising from the pelvis. Alphafetoprotein (AFP), $\beta$-human chorionic gonadotropin ( $\beta$-HCG) and lactate dehydrogenase $(\mathrm{LDH})$ were elevated. Bilateral salpingo-oopherectomy with infra-colic-omentectomy was performed. Histopathology revealed a left ovarian dysgerminoma and a right ovarian teratoma. Conclusion: In pediatric population, germ cell tumors are the commonest type. Concurrent tumors can be synchronous, independently derived, non-metastatic or metastatic. In adolescent patients, a timely diagnosis is essential as bilateral ovarian tumors pose challenges regarding ovarian conservation and reproductive future.
\end{abstract}

Keywords: Abdominal Lump, Ovarian Tumor, Dysgerminoma, Ovarian Teratoma, Pain.

\section{Introduction}

Ovarian neoplasms are uncommon in the pediatric age group, with an estimated incidence of 2.6 cases per 100,000 girls per year [1]. Ovarian malignancy is reported in $10-20 \%$ of all ovarian neoplasms occurring in children and adolescents comprising of approximately $1-2 \%$ of all childhood malignancies [2-4]. Ovarian malignancies in children and adolescents are rare as compared to adults but are the most common genital tumors, accounting for $60-70 \%$ of all gynecologic malignancies in this age group. Thus ovarian tumors constitute an important part of pediatric oncology and often create diagnostic dilemmas.

Ovarian tumors are well known for bilateral involvement. The likelihood of bilateral involvement by primary ovarian tumor varies with histological subtype. Very few case reports of synchronous bilateral primary ovarian tumors of different histologic subtypes have been described in literature. Here we report a rare case of a left ovarian dysgerminoma and a right ovarian teratoma in an 11 year old girl.

\section{Case Report}

An 11 year old girl presented with complaints of a lump in the lower abdomen since one month. The lump was associated with dull aching pain. There were no other associated symptoms. The girl had not attained menarche. On abdominal examination, a lump of size $15 \times 10 \mathrm{~cm}$ was palpable arising from the pelvis and occupying the entire abdomen. The lump was hard in consistency, non-tender and with minimal side to side mobility.

Ultrasonography of the abdomen and pelvis confirmed the clinical findings. Contrast enhanced computed tomography (CECT) abdomen showed a well-defined lobulated, solidcystic, heterogeneously enhancing mass lesion 
of approximate size $17 \times 16 \times 9 \mathrm{~cm}$ arising from the pelvis on the left side and extending upto the right hypochondrium. Few calcific areas, few fat attenuating as well as few non-enhancing necrotic areas were noted within the lesion. Left ovary was not visualized separately from the lesion. The uterus appeared normal. The right ovary was seen abutting the mass. Imaging features were suggestive of neoplastic etiology, possibility of left ovarian teratoma appeared more likely. Serological tumor markers including alpha-fetoprotein (AFP), $\beta$-human chorionic gonadotropin $(\beta-\mathrm{HCG})$ and lactate dehydrogenase (LDH) were elevated.

The patient underwent laparotomy. There was evidence of a bosselated mass of $18 \times 10 \mathrm{~cm}$ involving the left ovary [Fig.1]. It had both solid and cystic components. There was a similar mass of $8 \times 4 \mathrm{~cm}$ involving the right ovary [Fig.2]. The uterus, bowel, omentum and liver appeared normal. There was no obvious lymphadenopathy. A gynaecological opinion was taken. After counseling and with consent of the parents a bilateral salpingooopherectomy with infra-colic-omentectomy with retro-peritoneal biopsy was done. The ascitic fluid was collected and sent for cytological analysis.

The patient had no complications in the post-operative period and was discharged on post-operative day seven. The pathological report revealed a dysgerminoma of the left ovary and a mature cystic teratoma (dermoid cyst) of the right ovary. Lymph nodes, omentum and ascitic fluid were free of neoplastic involvement. The patient was referred to an oncologist and as post operatively the tumor markers were normal and no chemotherapy was given. Currently she is being followed up every three months without any evidence of disease.

\section{Discussion}

Ovarian tumors that occur in childhood and adolescence encompass a variety of benign and malignant tumors. The histological distributions

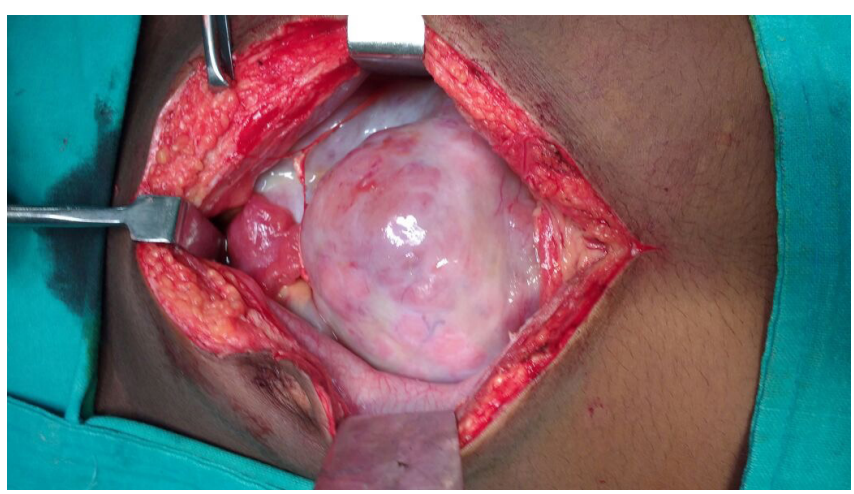

Fig.1: Intra-abdominal mass on exploration.

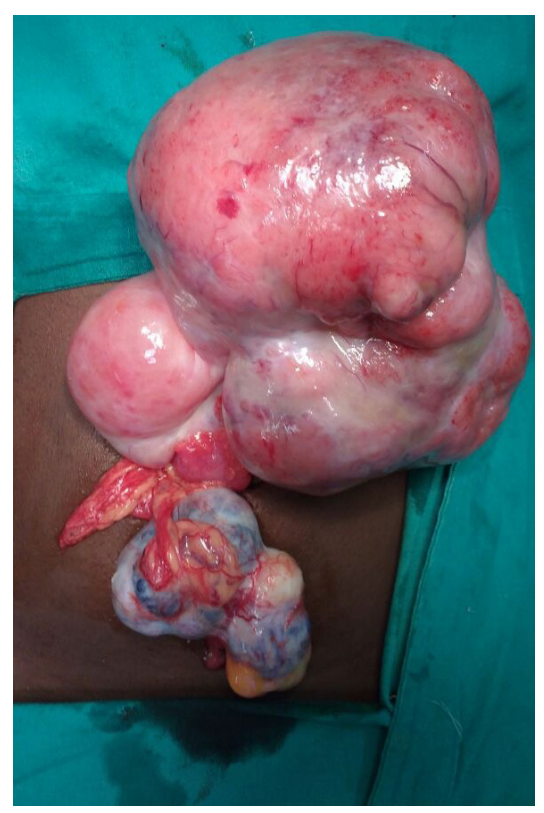

Fig.2: Bilateral ovarian mass.

of ovarian tumors differ substantially between pediatric and adult populations. Germ cell tumors $(60-80 \%)$ are the most common type of pediatric ovarian tumor, followed by surface epithelial stromal tumors $(15-20 \%)$ and sex cord stromal tumors $(10-20 \%)[5,6]$, whereas epithelial stromal tumors most commonly occur in adults.

Abdominal pain is the most common presenting symptom of ovarian tumors $(57 \%)$, followed by a palpable abdominal mass (46\%) [6]. Patients may also present with nausea, vomiting, poor appetite, weight loss, constipation and urinary 
frequency, or they may be asymptomatic, with the tumor being detected incidentally. Tenderness may be a sign of torsion, hemorrhage, or rupture of the ovarian tumor, especially GCTs and SCSTs. Children with a palpable mass or precocious puberty are reported to have a high likelihood of malignancy [4]. Acute onset of pain or tenderness caused by torsion appears to be a more frequent symptom of benign rather than malignant ovarian tumor.

In young girls with a suspected adnexal mass, the first diagnostic step is to define the exact site of origin and to distinguish it from other diseases with similar symptoms. Lesions with an ovarian origin should be determined to be either physiologic or neoplastic. The likelihood of malignancy should be assessed because this is critical when assigning patients to either conservative management or an appropriate surgical approach for fertility preservation.

Serum tumor markers are important in establishing the differential diagnosis [Table 1]. Among these tumor markers, elevated AFP, $\beta$-HCG and CA-125 levels raise concern for ovarian malignancy. However, negative tumor markers do not exclude the possibility of malignancy because the markers are positive in only $54 \%$ of cases [4]. Nevertheless, estimation of serum AFP, $\beta$-HCG levels is essential in children and adolescents who present with an adnexal mass.

Concurrent tumors are a well-known entity, at times posing diagnostic and therapeutic difficulties. They can be synchronous, independently derived, non-metastatic tumors or metastatic tumors. In general if tumors arising from different sites have different histologic features, they are generally regarded as independently derived primary tumors, which generally have a better prognosis than the primary tumor with metastasis [7].

A synchronous malignant tumor is defined as the occurrence of two tumor types within a six month period in the same patient. The occurrence of primary synchronous malignancies of the genital tract is rare, the incidence of which varies between $0.7-1.5 \%$. Independently primary tumors of the endometrium and ovary are the most commonly encountered synchronous tumors of the female genital tract. Very few case reports of bilateral synchronous primary ovarian malignant tumors have been mentioned in literature, as this case in which there was a left sided dysgerminoma with a right sided dermoid cyst.

Ovarian teratomas are GCTs composed of mature or immature tissues derived from more than one of the three primitive embryonic layers (ectoderm, mesoderm and endoderm). Mature cystic teratomas (MCT), often called dermoid cyst when the ectodermal elements predominate, is the most common ovarian tumor in children and adolescents, accounting for approximately 50\% of all pediatric ovarian neoplasms [8]. MCTs are bilateral in upto $10 \%$ of pediatric cases, and are typically asymptomatic. Optimal treatment of MCT in the pediatric population is surgical excision with fertility preservation; cystectomy and laparoscopic surgery are preferred for an uncomplicated MCT [8].

Dysgerminoma originates from undifferentiated germ cells and it is analogous to testicular seminoma. It is the most common malignant GCT in children and adolescence. Unlike other malignant GCTs, dysgerminoma occurs bilaterally in $10 \%-15 \%$ of cases and occasionally spreads to the retroperitoneal lymph nodes [8]. Therefore, the contralateral ovary and lymphatic spread should be assessed at diagnosis and followup. $\mathrm{LDH}$ is a useful tumor marker for diagnosis and post-operative follow-up. Most patients with dysgerminoma $(75 \%)$ are diagnosed with early stage disease, and thus surgery alone is curative [8]. The prognosis is usually excellent.

Bilateral ovarian tumors in a girl causes the dilemma of conservative versus aggressive approach towards these tumors. When faced with 
suspicious tumor and complete replacement of the ovaries bilaterally, bilateral oophorectomy is a viable option, though the certain possibility of infertility and lifelong hormonal supplementation is unavoidable.

\section{Conclusion}

The majority of pediatric ovarian tumors are benign, and the patients with malignant lesions have good prognosis. A timely diagnosis in cases of pelvic masses in the adolescent patient must be emphasized, in order to provide early and adequate treatment, thus causing the least possible impact on the reproductive future of these young women. Bilateral ovarian tumors pose management challenges with regards to ovarian conservation.

Contributors: MHA: manuscript writing, patient management; KPK: manuscript editing, patient management; KPK will act as guarantor. Both authors approved the final version of this manuscript.

Funding: None; Competing interests: None stated.

\section{References}

1. Skinner MA, Schlatter MG, Heifetz SA, Grosfeld JL. Ovarian neoplasms in children. Arch Surg. 1993;128(8):849-853; discussion 853-854.
2. Diamond MP, Baxter JW, Peerman CG Jr, Burnett LS. Occurrence of ovarian malignancy in childhood and adolescence: a community-wide evaluation. Obstet Gynecol. 1988;71(6 Pt 1):858-860.

3. Hassan E, Creatsas G, Deligeorolgou E, Michalas S. Ovarian tumors during childhood and adolescence: a clinicopathological study. Eur J Gynaecol Oncol 1999;20:124-126.

4. Oltmann SC, Garcia N, Barber R, Huang R, Hicks B, Fischer A. Can we preoperatively risk stratify ovarian masses for malignancy? J Pediatr Surg. 2010;45:130134.

5. Norris HJ, Jensen RD. Relative frequency of ovarian neoplasms in children and adolescents. Cancer. 1972;30:713-719.

6. Schultz KA, Sencer SF, Messinger Y, Neglia JP, Steiner ME. Pediatric ovarian tumors: a review of 67 cases. Pediatr Blood Cancer. 2005;44:167-173.

7. Brinkmann D, Ryan A, Ayhan A, McCluggage WG, Feakins R, Santibanez-Koref MF, et al. A molecular genetic and statistical approach for the diagnosis of dualsite cancers. J Natl Cancer Inst. 2004;96:1441-1446.

8. Laufer MR, Goldstein DP. Benign and malignant ovarian masses. In: Emans SJH, Laufer MR, Goldstein DP, eds. Pediatric and adolescent gynecology. $5^{\text {th }}$ ed. Philadelphia, Pa: Lippincott Williams \& Wilkins, 2005; pp. 685. 